\title{
Interference Effects In A Photonic Crystal Cavity
}

P.M. Alsing and D.A. Cardimona

20 January 2010

Interim Report

\author{
AIR FORCE RESEARCH LABORATORY \\ Space Vehicles Directorate \\ 3550 Aberdeen Ave SE \\ AIR FORCE MATERIEL COMMAND \\ KIRTLAND AIR FORCE BASE, NM 87117-5776
}




\section{DTIC COPY}

\section{NOTICE AND SIGNATURE PAGE}

Using Government drawings, specifications, or other data included in this document for any purpose other than Government procurement does not in any way obligate the U.S. Government. The fact that the Government formulated or supplied the drawings, specifications, or other data does not license the holder or any other person or corporation; or convey any rights or permission to manufacture, use, or sell any patented invention that may relate to them.

Qualified requestors may obtain copies of this report from the Defense Technical Information Center (DTIC) (http://www.dtic.mil).

\section{AFRL-RV-PS-TP-2010-1001 HAS BEEN REVIEWED AND IS APPROVED FOR PUBLICATION IN ACCORDANCE WITH ASSIGNED DISTRIBUTION STATEMENT.}

//SIGNED//

DAVID CARDIMONA

Program Manager
//SIGNED//

WILLIAM A. SCHUM, Lt Col, USAF

Deputy Chief, Spacecraft Technology Division

Space Vehicles Directorate

This report is published in the interest of scientific and technical information exchange, and its publication does not constitute the Government's approval or disapproval of its ideas or findings. 


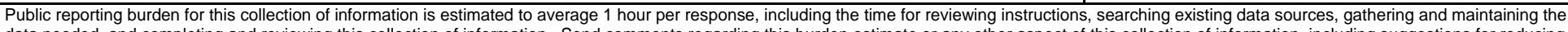

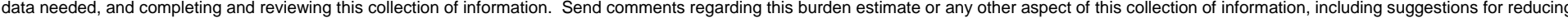

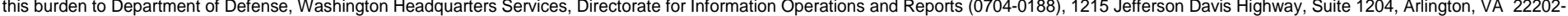

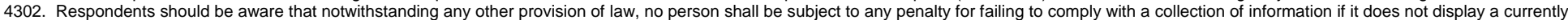
valid OMB control number. PLEASE DO NOT RETURN YOUR FORM TO THE ABOVE ADDRESS.

\begin{tabular}{l|l|l} 
1. REPORT DATE (DD-MM-YYYY) & 2. REPORT TYPE & 3. DATES COVERED (FrOm - To)
\end{tabular}

$20 / 01 / 2010$

Interim Report

4. TITLE AND SUBTITLE

Interference Effects In A Photonic Crystal Cavity

09/01/2008 - 07/01/2010

5a. CONTRACT NUMBER

5b. GRANT NUMBER

5c. PROGRAM ELEMENT NUMBER

$62601 \mathrm{~F}$

6. AUTHOR(S)

P.M. Alsing, D.A. Cardimona

\section{5d. PROJECT NUMBER}

4846

5e. TASK NUMBER

CR

5f. WORK UNIT NUMBER

406527

\section{PERFORMING ORGANIZATION NAME(S) AND ADDRESS(ES)}

8. PERFORMING ORGANIZATION REPORT NUMBER

Air Force Research /aboratory

AFRL-RV-PS-TP-2010-1001

Space Vehicles Directorate

3550 Aberdeen Ave., SE

Kirtland AFB, NM 87117-5776

9. SPONSORING / MONITORING AGENCY NAME(S) AND ADDRESS(ES)

10. SPONSOR/MONITOR'S ACRONYM(S)

AFRL/RVSS

11. SPONSOR/MONITOR'S REPORT NUMBER(S)

12. DISTRIBUTION / AVAILABILITY STATEMENT

Approved for Public Release; Distribution is Unlimited. (Clearance \#377 ABW-2009-1628)

\section{SUPPLEMENTARY NOTES}

Government Purpose Rights. Proceedings of SPIE Quantum Sensing and Nanophotonic Devices VII vol. 7608, pp 760817-1-11 (2010) 23 Dec 09

\section{ABSTRACT}

At the Space Vehicles Directorate of the Air Force Research Laboratory we are interested in the use of detectors in space for surveillance and situational awareness missions. Our primary interests are in observations of objects both on earth and in space, each of which has very different background requirements. In addition, the space environment itself is especially demanding of any sensor system that will be expected to work continuously for long periods of time in such a challenging environment. In this talk we investigate quantum interference and classical interference effects when a three-level system interacts with both a cavity field mode and an external driving field mode, within the confines of a photonic crystal material.

\section{SUBJECT TERMS}

Quantum interference, photonic crystals, cavity quantum electrodynamics

\begin{tabular}{|c|c|c|c|c|c|}
\hline 16. SECURITY CLAS & IFICATION OF: & & $\begin{array}{l}\text { 17. LIMITATION } \\
\text { OF ABSTRACT }\end{array}$ & $\begin{array}{l}\text { 18. NUMBER } \\
\text { OF PAGES }\end{array}$ & $\begin{array}{l}\text { 19a. NAME OF RESPONSIBLE PERSON } \\
\text { David Cardimona }\end{array}$ \\
\hline $\begin{array}{l}\text { a. REPORT } \\
\text { Unclassified }\end{array}$ & $\begin{array}{l}\text { b. ABSTRACT } \\
\text { Unclassified }\end{array}$ & $\begin{array}{l}\text { c. THIS PAGE } \\
\text { Unclassified }\end{array}$ & Unlimited & 16 & $\begin{array}{l}\text { 19b. TELEPHONE NUMBER (include area } \\
\text { code) }\end{array}$ \\
\hline
\end{tabular}


(This page intentionally left blank) 


\title{
Interference Effects in a Photonic Crystal Cavity
}

\author{
D. A. Cardimona and P. M. Alsing* \\ Air Force Research Laboratory, Space Vehicles Directorate, 3550 Aberdeen Ave., \\ Kirtland AFB, NM 87117
}

\begin{abstract}
We investigate quantum interference and classical interference effects when a three-level system interacts with both a cavity field mode and an external driving field mode, within the confines of a photonic crystal material. In freespace, we found that under certain circumstances the cavity field evolves to be equal in magnitude to, but $180^{\circ}$ outof-phase with the external pump field when the pump field frequency equals the cavity frequency. The better the cavity, the quicker this build-up occurs. When the cavity field reaches this out-of-phase condition, the resonance fluorescence from the atom in the cavity goes to zero. This is a purely classical interference effect between the two out-of-phase fields, with the resonance fluorescence going to zero at the same time as the two excited state populations go to zero. This is quite different from the quantum interference that occurs under the right circumstances, when the state populations are coherently driven into a linear combination that is decoupled from any applied field - and population is trapped in the excited states, thus allowing for a population inversion and an amplification of incoming optical signals. In this paper, we investigate the additional effects due to the presence of the altered photon density of states in a photonic crystal.
\end{abstract}

Key Words: quantum interference, photonic crystals, cavity quantum electrodynamics

\section{INTRODUCTION}

Three-level systems have the ability to offer quantum interference effects due to the multiple transitions available, thereby allowing the spontaneous emission from such a system to be controlled. These quantum interference effects often result in coherent population trapping, which can be used for lasing without inversion or optical signal amplification. Quantum interference leading to field-induced transparency (FIT) was first described by Cardimona et al. in 1982 [1] for a system having two closely-spaced excited states each dipole-coupled to a common ground state(the so-called "V" system [see Fig. 1]). In FIT, an applied field tuned appropriately dresses the atom in such a way as to produce a dressed state that has a net-zero dipole moment. When this dressed atom undergoes spontaneous emission, the other two dressed states decay into the dipole-decoupled state, which in turn does not decay. Thus, population is trapped in this decoupled dressed state, and spontaneous emission ceases.

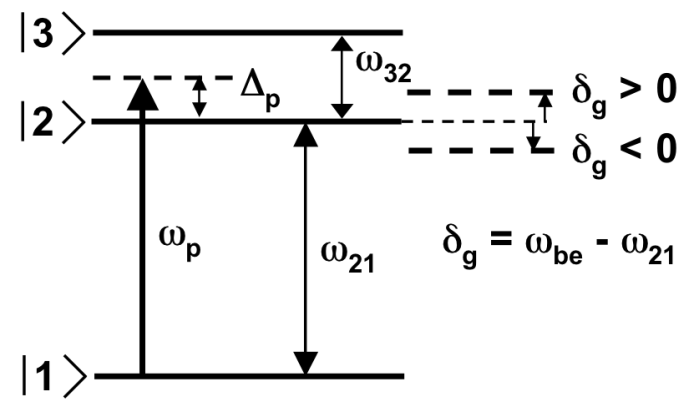

FIG. 1. Quantized system considered in this paper. This is a V-type atom with two closely spaced excited states, each dipole coupled to a common ground state, with a pump field tuned between the two excited states. Here, $\omega_{\mathrm{p}}$ is the pump frequency, detuned from the 2-to- 1 transition by $\Delta_{\mathrm{p}}$, and $\delta_{\mathrm{g}}$ is the tuning of the photonic crystal band edge frequency $\left(\omega_{\text {be }}\right)$ from the 2-to-1 transition frequency.

*Now at The Information Directorate, Rome, NY 13441 
In 1989, Harris described a similar effect in the so-called " $\Lambda$ " system, which he called electromagnetically induced transparency (EIT) [2], in which a lower excited state is coupled to a higher excited state with a control field, and then the higher excited state is probed with another field coupling that excited state to the ground state. In this case, the control field dresses the system so that the excited state Rabi splits into an Autler-Townes doublet via the ac Stark effect. The probe field then sees a system very similar to the FIT case, with the two excited states now being the ac-Stark-split doublet and experiencing the same quantum intereference as described above.

In this paper we investigate quantum interference and classical-interference effects when quantized three-level systems (e.g., atoms, quantum dots, semiconductor quantum well heterostructures) in a photonic crystal (PhC) cavity interact with both a cavity-field mode and an external driving field mode. In Ref. [3], we found that under certain circumstances the cavity field evolved to be equal in magnitude to, but $180^{\circ}$ out of phase with, the external pump field when the pump-field frequency was equal to the cavity frequency. The better the cavity, the quicker this buildup occurred. When the cavity field reached this "out-of-phase" condition, the resonance fluorescence from the atom in the cavity went to zero. This was a purely classical-interference effect between the two out-of-phase fields, with the resonance fluorescence (proportional to the susceptibility of the system) going to zero at the same time as the two excited state populations go to zero. This is quite different from the quantum interference that occurs under the right circumstances when the state populations are coherently driven into a linear combination that is decoupled from any applied field, and population is trapped in the excited states.

In this paper we investigate how the effects of a PhC medium will affect these results (see Ref. [4] for an investigation of quantum interference near a PhC band edge). In a series of papers during the 1990s, John and collaborators [5], introduced a simple model of a 3D periodic dielectric material with an exact analytical expression (in terms of a transcendental equation) for the photon dispersion relation [6]. In the vicinity of the photonic band edge (PBE), the dispersion relation can be expanded in a Taylor series to second order in wavevector, without a linear contribution, resulting in:

$$
\omega_{k}=\omega_{b e}+\frac{c^{2}}{\omega_{g}}\left(k-\frac{\omega_{g}}{c}\right)^{2},
$$

where $\omega_{b e}$ is the band edge frequency of the PBG and $\omega_{g}$ is the frequency width of the bandgap. In this model, the presence of the PBE modifies the density of states from that of the usual free space form of $\rho(\omega) \sim \omega^{2}$ to

$$
\rho(\omega) \sim \frac{\Theta\left(\omega-\omega_{b e}\right)}{\sqrt{\omega-\omega_{b e}}},
$$

(see Fig. 2) where $\Theta\left(\omega-\omega_{b e}\right)$ is the Heaviside step function (which equals 1 for $\left(\omega-\omega_{b e}\right)>0$ and equals 0 elsewhere).

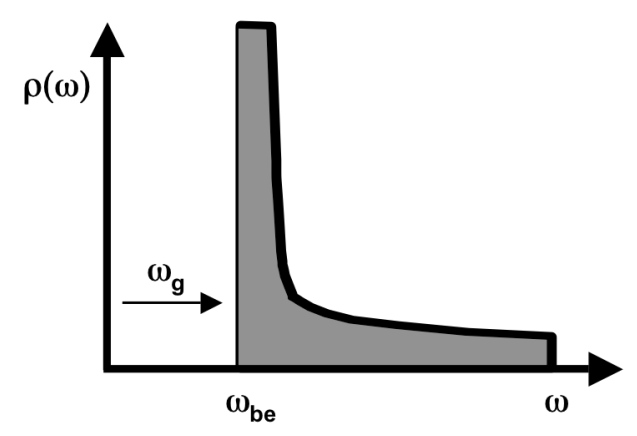

FIG. 2. Density of states [Eq. (2)] for the dispersion relation in Eq. (1), valid near the PBE of frequency $\omega_{b e}$ and PBG of width $\omega_{g}$. 
In this work we present a straightforward density matrix approach which allows us to analyze spontaneous emission and quantum interference effects in the vicinity of a PBE for arbitrary probe and driving field strengths. We develop the density matrix equations by returning to the well-developed theory of spontaneous emission in the Heisenberg picture and rearranging the formulation so that the non-Markovian convolution integrals between the atomic and field degrees of freedom can be dealt with beyond the Wigner-Weisskopf pole approximation for the model of the PBG introduced by John and co-workers and discussed above.

\section{GENERAL FORMULATION FOR A MULTI-LEVEL SYSTEM IN A PHOTONIC CRYSTAL CAVITY}

The Hamiltonian for the system under consideration, an atom interacting with multiple fields, is the sum of the atom, field, and interaction Hamiltonians [see Ref. 3]:

$$
\hat{H}=\hat{H}_{a}+\hat{H}_{f}+\hat{H}_{\mathrm{int}}
$$

where

$$
\begin{aligned}
& \hat{H}_{a}=\sum_{j} \hbar \omega_{j} \widehat{\sigma}_{j j}(t) \\
& \widehat{H}_{f}=\sum_{\vec{k}, s} \hbar \omega_{k} \widehat{a}_{\vec{k} s}(t) \widehat{a}_{\vec{k} s}^{+}(t)=\hbar \omega_{p} \widehat{a}_{p}(t) \widehat{a}_{p}^{+}(t)+\hbar \omega_{c} \widehat{a}_{c}(t) \widehat{a}_{c}^{+}(t) \\
& +\sum_{\vec{k} \neq \vec{k}_{c}, s} \hbar \omega_{k} b_{\vec{k} s}(t) \hat{b}_{\vec{k} s}^{+}(t)+\sum_{\hat{k}_{c}, s} \hbar \omega_{k_{c}} \widehat{c}_{\vec{k}_{c} s}(t) \hat{c}_{\vec{k}_{c} s}^{+}(t), \\
& \widehat{H}_{\mathrm{int}}=-\underline{\hat{d}}(t) \bullet\left(\underline{\hat{E}}_{p}(t)+\underline{\hat{E}}_{c}(t)+\underline{\hat{E}}_{s p}(t)\right)+\frac{1}{2} \varepsilon v_{c} T_{c} \underline{\hat{E}}_{c}(t) \bullet \underline{\hat{E}}_{c_{s p}}(t) .
\end{aligned}
$$

Here $\widehat{\sigma}_{j j}$ are the Heisenberg-picture atomic state population operators, $\hat{a}_{\vec{k} s}\left(\hat{a}_{\vec{k} s}^{+}\right)$are the field raising (lowering) operators (with $k, \mathrm{~s}=\mathrm{p}$ for the single-mode external pump field, $k, s=c$ for the single-mode internal cavity field, $\widehat{a}_{\vec{k} s}=\widehat{b}_{\vec{k} s}$ for the spontaneous field modes emitted by the atom that leave out the sides of the cavity, and $\widehat{a}_{\vec{k} s}=\widehat{c}_{\vec{k}_{c} s}$ for the spontaneous field modes that leak out through the mirrors of the cavity), $\underline{\hat{d}}$ is the atomic dipole moment operator, $\underline{E}_{p}$ is the external pump field operator, $\underline{E}_{c}$ is the cavity field operator, $\underline{\hat{E}}_{s p}$ is the operator for the spontaneous field emitted by the atom (atomic resonance fluorescence), $\varepsilon$ is the permittivity of the materials within the cavity, $v_{c}$ is the volume of the cavity, $T_{c}$ is the transmissivity of the cavity mirrors, $\underline{\underline{E}}_{c_{s p}}$ is the cavity leakage field operator (cavity fluorescence). The field operators are given by:

$$
\begin{aligned}
& \underline{\underline{E}}_{p}(t)=i \sqrt{\frac{2 \pi \hbar \omega_{p}}{V_{p}}}\left(\underline{\varepsilon}_{p} \hat{a}_{p}(t)-\underline{\varepsilon}_{p}^{*} \hat{a}_{p}^{+}(t)\right), \\
& \underline{\hat{E}}_{c}(t)=i \sqrt{\frac{2 \pi \hbar \omega_{c}}{v_{c}}}\left(\underline{\varepsilon}_{c} \hat{a}_{c}(t)-\underline{\varepsilon}_{c}^{*} \hat{a}_{c}^{+}(t)\right), \\
& \underline{\hat{E}}_{s p}(t)=i \sum_{\vec{k} \neq \vec{k}_{c}, s} \sqrt{\frac{2 \pi \hbar \omega_{k}}{V_{k}}}\left(\underline{\varepsilon}_{\vec{k} s} \hat{b}_{\vec{k} s}(t)-\underline{\varepsilon}_{\vec{k} s}^{*} \hat{b}_{\vec{k} s}^{+}(t)\right),
\end{aligned}
$$




$$
\underline{\underline{E}}_{c_{s p}}(t)=i \sum_{\vec{k}_{c}, s} \sqrt{\frac{2 \pi \hbar \omega_{k_{c}}}{V_{k_{c}}}}\left(\underline{\varepsilon}_{\vec{k}_{c}} \vec{c}_{\vec{k}_{c} s}(t)-\underline{\varepsilon}_{\vec{k}_{c} s}^{*} \widehat{c}_{\vec{k}_{c} s}^{+}(t)\right),
$$

where $V_{p}, V_{k}$, and $V_{k_{c}}$ are the quantization volumes for the pump, atomic fluorescence field, and the cavity leakage field, respectively. Here the complex field polarization vectors are represented by $\underline{\varepsilon}_{p}, \underline{\varepsilon}_{c}, \underline{\varepsilon}_{\vec{k} s}$, and $\underline{\varepsilon}_{\vec{\varepsilon}_{c} s}$, again for the pump, cavity, atomic fluorescence, and cavity leakage fields. The dipole moment operator is given by:

$$
\underline{\hat{d}}(t)=\sum_{i, j} \underline{d}_{i j} \widehat{\sigma}_{i j}(t),
$$

with $\underline{d}_{i j}$ equal to the transition dipole moment between atomic states $|i\rangle$ and $|j\rangle$ and $\widehat{\sigma}_{i j}$ are the Heisenberg-picture atomic transition operators.

For the time dynamics of this system, we find the time derivative of any operator $\hat{X}(t)$ by using the commutation relation:

$$
\frac{d \widehat{X}(t)}{d t}=\frac{1}{i \hbar}[\widehat{X}(t), \hat{H}]
$$

Further noting that $\left[\hat{a}_{\vec{k} s}, \hat{a}_{\vec{k}^{\prime} s^{\prime}}^{+}\right]=\delta_{\vec{k} s, \vec{k}^{\prime} s^{\prime}}^{4}$, we find the following dynamic equations:

$$
\begin{aligned}
& \frac{d \widehat{\sigma}_{i j}(t)}{d t}=i \omega_{i j} \widehat{\sigma}_{i j}(t)-\sum_{\vec{k} \neq \vec{k}_{c}, s} \sqrt{\frac{2 \pi \omega_{k}}{\hbar V_{k}}} \sum_{n}\left[\underline{d} \text { jn } \bullet\left(\underline{\varepsilon}_{\vec{k} s} \widehat{\sigma}_{i n}(t) \widehat{a}_{\vec{k} s}-\underline{\varepsilon}_{\vec{k} s}^{*} \hat{a}_{\vec{k} s}^{+} \widehat{\sigma}_{i n}(t)\right)\right. \\
& \left.-\underline{d}_{n i} \bullet\left(\underline{\varepsilon}_{\vec{k} s} \widehat{\sigma}_{n j}(t) \hat{a}_{\vec{k} s}-\underline{\varepsilon}_{\vec{k} s}^{*} \hat{a}_{\vec{k} s}^{+} \widehat{\sigma}_{n j}(t)\right)\right], \\
& \frac{d \widehat{a}_{p}(t)}{d t}=-i \omega_{p} \widehat{a}_{p}(t) \quad\left(\Rightarrow \widehat{a}_{p}(t)=\widehat{a}_{p}(0) e^{-i \omega_{p} t}\right), \\
& \frac{d \bar{b}_{\vec{k} s}(t)}{d t}=-i \omega_{k} \widehat{b}_{\vec{k} s}(t)+\sqrt{\frac{2 \pi \omega_{\mathrm{k}}}{\hbar \mathrm{V}_{\mathrm{k}}}} \underline{d}(t) \bullet \underline{\varepsilon}_{\vec{k} s}^{*}, \\
& \frac{d \widehat{a}_{c}(t)}{d t}=-i \omega_{c} \widehat{a}_{c}(t)+\sqrt{\frac{2 \pi \omega_{\mathrm{c}}}{\hbar v_{\mathrm{c}}}} \underline{\underline{d}}(t) \bullet \underline{\varepsilon}_{c}^{*} \\
& -i \frac{1}{2} \varepsilon v_{c} T_{c} \sqrt{\frac{2 \pi \hbar \omega_{c}}{v_{c}}} \sum_{\vec{k}_{c}, s} \sqrt{\frac{2 \pi \omega_{k_{c}}}{\hbar V_{c}}}\left(\underline{\varepsilon}_{c}^{*} \bullet \underline{\varepsilon}_{k_{c}} \vec{c}_{\vec{k}_{c} s}(t)-\underline{\varepsilon}_{c}^{*} \bullet \underline{\varepsilon}_{\vec{k}_{c}}^{*} \widehat{c}_{\vec{k}_{c} s}^{+}(t)\right), \\
& \frac{d \widehat{c}_{\vec{k}_{c} s}(t)}{d t}=-i \omega_{k_{c}} \widehat{c}_{\vec{k}_{c} s}(t)-\mathrm{i} \frac{1}{2} \varepsilon v_{c} T_{c} \sqrt{\frac{2 \pi \hbar \omega_{c}}{v_{c}}} \sqrt{\frac{2 \pi \omega_{k_{c}}}{\hbar V_{k_{c}}}}\left(\underline{\varepsilon}_{c} \bullet \underline{\varepsilon}_{k_{c} s}^{*} \widehat{a}_{c}(t)-\underline{\varepsilon}_{c}^{*} \bullet \underline{\varepsilon}_{k_{c}}^{*} \vec{a}_{c}^{+}(t)\right) .
\end{aligned}
$$

Note that in Eq. (14) we treat the pump as a constant classical external field (anticipating making the pump a coherent state), and therefore drop the $\left[\widehat{a}_{p}(t), \hat{H}_{\text {int }}\right]$ term. In this approximation we assume that the atom adding a photon or two to the classical pump will not change it. The interaction of the pump on the atom is still accounted for in Eq. (13) in the complete sum over all field modes $k, s$.

Formally solving the two spontaneous field equations (15) and (17) results in: 


$$
\begin{aligned}
& \hat{b}_{\vec{k} s}(t)=\sqrt{\frac{2 \pi \omega_{\mathrm{k}}}{\hbar \mathrm{V}_{\mathrm{k}}}} \sum_{\substack{i, j \\
j>i}} \underline{d}_{i j} \bullet \underline{\varepsilon}_{\vec{k}}^{*} \int_{0}^{t} d t^{\prime} e^{-i \omega_{k}\left(t-t^{\prime}\right)} \hat{\sigma}_{i j}\left(t^{\prime}\right) \\
& \hat{c}_{\vec{k}_{c} s}(t)=-\mathrm{i} \frac{1}{2} \varepsilon v_{c} T_{c} \sqrt{\frac{2 \pi \hbar \omega_{\mathrm{c}}}{v_{\mathrm{c}}}} \sqrt{\frac{2 \pi \omega_{k_{c}}}{\hbar V_{k_{c}}}} \underline{\varepsilon}_{c} \cdot \underline{\varepsilon}_{\vec{\varepsilon}_{c} s}^{*} \int_{0}^{t} d t^{\prime} e^{-i \omega_{k_{c}}\left(t-t^{\prime}\right)} \widehat{a}_{c}\left(t^{\prime}\right)
\end{aligned}
$$

where we have dropped rapidly oscillating terms in $\bar{c}_{\vec{k}_{c} s}(t)$ [in anticipation of invoking the Rotating Wave Approximation (RWA) in the future]. Substituting Eq. (19) back into Eq. (16), and using the adiabatic approximation for $\widehat{a}_{c}\left(t^{\prime}\right)$,

$$
\widehat{a}_{c}\left(t^{\prime}\right) \approx \widehat{a}_{c}(t) e^{-i \omega_{c}\left(t-t^{\prime}\right)}
$$

in the integral that arises on the right side of the resulting equation, we find (with the reminder that the $\hat{a}_{c}^{+}(t)$ term has been dropped in the RWA):

$$
\frac{d \hat{a}_{c}(t)}{d t}=-i \omega_{c} \hat{a}_{c}(t)+\sqrt{\frac{2 \pi \omega_{\mathrm{c}}}{\hbar v_{\mathrm{c}}}} \sum_{i, j} \underline{d}_{i j} \bullet \underline{\varepsilon}_{c}^{*} \widehat{\sigma}_{i j}(t)-\int_{0}^{t} d t^{\prime} K_{c}\left(t-t^{\prime}\right) \hat{a}_{c}(t),
$$

where

$$
\begin{aligned}
& K_{c}\left(t-t^{\prime}\right) \equiv\left(\frac{1}{2} \varepsilon v_{c} T_{c}\right)^{2} \frac{2 \pi \hbar \omega_{\mathrm{c}}}{v_{\mathrm{c}}} \sum_{\vec{k}_{c}, s} \frac{2 \pi \omega_{k_{c}}}{\hbar V_{k_{c}}}\left|\underline{\varepsilon}_{c} \bullet \underline{\varepsilon}_{k_{c} s}^{*}\right|^{2} e^{-i\left(\omega_{k_{c}}-\omega_{c}\right)\left(t-t^{\prime}\right)} \\
& \underset{V_{k_{c}} \rightarrow \infty}{\longrightarrow}\left\{\begin{array}{l}
\left.\left(\frac{1}{2} \varepsilon v_{c} T_{c}\right)^{2} \frac{2 \pi \hbar \omega_{\mathrm{c}}}{v_{\mathrm{c}}}\left[\frac{2}{3 \hbar c^{3}}\left|\underline{\varepsilon}_{c}\right|^{2} \mid \omega_{c}^{3}-i \frac{1}{\pi} P \int \frac{\omega^{3} d \omega}{\omega-\omega_{c}}\right)\right] \delta\left(t-t^{\prime}\right), \rho(\omega) \sim \omega^{2} \\
\left(\frac{1}{2} \varepsilon v_{c} T_{c}\right)^{2} \frac{2 \pi \hbar \omega_{\mathrm{c}}}{v_{\mathrm{c}}}\left[\frac{\omega_{c}^{2} \omega_{b e} \sqrt{\omega_{\mathrm{g}}}\left|\underline{\varepsilon}_{c}\right|^{2}}{12 \pi^{3 / 2} \varepsilon \hbar c^{3}} \frac{e^{-i\left[\pi / 4-\delta_{c}\left(t-t^{\prime}\right)\right]}}{\sqrt{t-t^{\prime}}}\right], \rho(\omega) \sim \frac{\Theta\left(\omega-\omega_{b e}\right)}{\sqrt{\omega-\omega_{b e}}},
\end{array}\right.
\end{aligned}
$$

with $\delta_{c}=\omega_{b e}-\omega_{c}$ and "P" stands for "principal part" integral. For the free-space density of states result, the first term is the cavity spontaneous decay constant $(\kappa)$, and the second (imaginary) term is the cavity "Lamb shift" ( $S_{c a v}$, which we will ignore in this work).

We note that the adiabatic approximation assumes that the atom-field interaction is weak. Formally this approximation is invalid near the photonic band edge $\omega_{b e}$ where the density of states diverges. However, we can avoid this divergence by introducing a level-broadening parameter based on the long, but constant average finite lifetime $\tau$ of the field modes in any realistic PhC. The density of states would then be given by

$$
\rho(\omega) \sim\left\{\begin{array}{l}
\frac{1}{\sqrt{\omega-\omega_{b e}}}, \omega>\omega_{b e}+1 / \tau \\
\sqrt{\tau}, \omega_{b e}<\omega<\omega_{b e}+1 / \tau
\end{array} .\right.
$$

The constant, finite density of states for $\omega_{b e}<\omega<\omega_{b e}+1 / \tau$ allows us to invoke the adiabatic approximation. [4] In the following we ignore this technical detail for the most part and treat $\tau$ as infinite, since all sums over modes and integrals over time can be computed exactly in this limit. 
Now we formally integrate Eq. (21) and note that in the RWA for the FIT atom in Fig. 1, only terms that oscillate like $e^{-i \omega_{p} t}\left(\right.$ since $\omega_{p} \approx \omega_{c}$ ) will survive. Therefore, in anticipation of invoking the RWA later, we use the adiabatic approximation for the atomic transition operators in the form

$$
\widehat{\sigma}_{i j}\left(t^{\prime}\right) \approx \widehat{\sigma}_{i j}(t) e^{-i \omega_{p}\left(t-t^{\prime}\right)}
$$

to obtain

$$
\hat{a}_{c}(t) \approx \sqrt{\frac{2 \pi \omega_{\mathrm{c}}}{\hbar v_{\mathrm{c}}}} \sum_{\substack{i, j \\ j>i}} \underline{d}_{i j} \bullet \underline{\varepsilon}_{c}^{*} \widehat{\sigma}_{i j}(t) \frac{1}{\bar{K}_{c}\left(\delta_{c}\right)+i\left(\omega_{c}-\omega_{p}\right)},
$$

where $\hat{a}_{c}(0)=0$ and

$$
\begin{aligned}
\bar{K}_{c}\left(\delta_{c}\right) & =\lim _{t \rightarrow \infty} \int_{0}^{t} d t^{\prime} K_{c}\left(t-t^{\prime}\right), \\
& =\left\{\begin{array}{cc}
-i \frac{\omega_{c}^{2} \omega_{b e} \sqrt{\omega_{\mathrm{g}}}\left|\underline{\varepsilon}_{c}\right|^{2}}{12 \pi^{3 / 2} \varepsilon \hbar c^{3}} \frac{1}{\sqrt{\left|\delta_{c}\right|}}+\Gamma_{P h C} \equiv-i \frac{\beta_{c}}{\sqrt{\left|\delta_{c}\right|}}+\Gamma_{P h C}, \delta_{c}>0 \text { or } \omega_{b e}>\omega_{c} \\
\frac{\beta_{c}}{\sqrt{\left|\delta_{c}\right|}}, \delta_{c}<0 \text { or } \omega_{b e}<\omega_{c}
\end{array}\right.
\end{aligned}
$$

Recall that we are ignoring the finite lifetime $\tau$ of the PhC field modes, so we will stay away from the $\omega_{b e}=\omega_{c}$ limit; thus $\beta_{c} / \sqrt{\left|\delta_{c}\right|}$ will not diverge. The $\Gamma_{P h C}$ term when $\delta_{c}>0$ is phenomenologically inserted to take into account the cavity damping due to the finite lifetime $\tau\left(\Gamma_{P h C} \propto 1 / \tau\right)$. In order to continue to ignore the effects of the finite $\mathrm{PhC}$ lifetime, in this work we will only consider $\omega_{b e}<\omega_{c}$. For $\bar{K}_{c}\left(\delta_{c}\right)$ we have anticipated going to the steady-state limit.

Substituting these solutions back into Eq. (13) for the atomic transition operators, taking expectation values of these transition operators to get the density matrix elements $\left(\left\langle\widehat{\sigma}_{i j}(t)\right\rangle=\operatorname{Tr}\left[\hat{\rho}(t) \hat{\sigma}_{i j}(t)\right]=\rho_{j i}(t)\right)$, assuming the external pump field is in a coherent state, and throwing out terms that will be dropped when we take the RWA, we find:

$$
\begin{aligned}
\frac{d \rho_{i j}}{d t}=-i \omega_{i j} \rho_{i j}+\frac{i}{\hbar} \sum_{n}\left(\underline{d}_{i n} \rho_{n j}-\underline{d}_{n j} \rho_{i n}\right) \bullet \underline{E}_{p} \\
-\sum_{\substack{m, n \\
m>n}}\left[\bar{\Gamma}_{i n n m} \rho_{m j}+\bar{\Gamma}_{j n n m}^{*} \rho_{i m}\right]+\sum_{\substack{m, n \\
m>j}} \bar{\Gamma}_{n i j m}^{*} \rho_{n m}+\sum_{\substack{m, n \\
m>i}} \bar{\Gamma}_{n j i m} \rho_{m n},
\end{aligned}
$$

where $\underline{E}_{p}=i \varepsilon_{p}\left(\underline{\varepsilon}_{p} e^{-i \omega_{p} t}-\underline{\varepsilon}_{p}^{*} e^{i \omega_{p} t}\right)$ and the decay or frequency-shift (depending on the position of the band edge) terms are

$$
\bar{\Gamma}_{k l m n}=\bar{\gamma}_{k l m n}+\bar{\gamma}_{c_{k l m n}},
$$

with

$$
\bar{\gamma}_{k l m n}=\lim _{t \rightarrow \infty} \int_{0}^{t} d t^{\prime} K_{k l m n}\left(t-t^{\prime}\right)
$$




$$
\begin{gathered}
=\left\{\begin{array}{r}
-i \frac{\left|\omega_{k l}\right|\left|\omega_{m n}\right| \omega_{b e} \sqrt{\omega_{\mathrm{g}}}\left(\underline{d}_{k l} \bullet \underline{d}_{m n}\right)}{12 \pi^{3 / 2} \varepsilon \hbar c^{3}} \frac{1}{\sqrt{\left|\delta_{n m}\right|}} \equiv-i \beta_{k l m n}, \delta_{n m}>0 \text { or } \omega_{b e}>\omega_{n m} \\
\beta_{k l m n}, \delta_{n m}<0 \text { or } \omega_{b e}<\omega_{n m}
\end{array}\right. \\
K_{k l m n}\left(t-t^{\prime}\right) \equiv \lim _{V_{k} \rightarrow \infty} \sum \frac{2 \pi \omega_{k}}{\hbar V_{k}}\left(\underline{d}_{k l} \bullet \underline{\varepsilon}_{\vec{k} s}\right)\left(\underline{d}_{m n} \bullet \underline{\varepsilon}_{\vec{k} s}^{*}\right) e^{-i\left(\omega_{k}+\omega_{m n}\right)\left(t-t^{\prime}\right)} \\
=\left\{\begin{array}{c}
{\left[\frac{2}{3 \hbar c^{3}}\left(\underline{d}_{k l} \bullet \underline{d}_{m n}\right)\left(\omega_{n m}^{3}-i \frac{1}{\pi} P \int \frac{\omega^{3} d \omega}{\omega-\omega_{n m}}\right)\right] \delta\left(t-t^{\prime}\right), \rho(\omega) \sim \omega^{2}} \\
{\left[\frac{\left|\omega_{k l}\right|\left|\omega_{m n}\right| \omega_{b e} \sqrt{\omega_{\mathrm{g}}}\left(\underline{d}_{k l} \bullet \underline{d}_{m n}\right)}{12 \pi^{3 / 2} \varepsilon \hbar c^{3}} \frac{e^{-i\left[\pi / 4+\left(\omega_{b e}-\omega_{n m}\right)\left(t-t^{\prime}\right)\right]}}{\sqrt{t-t^{\prime}}}\right], \rho(\omega) \sim \frac{\Theta\left(\omega-\omega_{b e}\right)}{\sqrt{\omega-\omega_{b e}}}}
\end{array}\right.
\end{gathered}
$$

with $\delta_{n m}=\omega_{b e}-\omega_{n m}$, and all of the effects of the cavity are accounted for in

$$
\begin{aligned}
& \bar{\gamma}_{c_{k l m n}}=\frac{g_{k l} g_{n m}^{*}}{\bar{K}_{c}\left(\delta_{c}\right)+i\left(\omega_{c}-\omega_{p}\right)}, \\
& g_{i j} \equiv \sqrt{\frac{2 \pi \omega_{c}}{\hbar v_{c}}} \underline{d}_{i j} \bullet \underline{\varepsilon}_{c} .
\end{aligned}
$$

Once again, the "principal part" integral in Eq. (30b) is the natural Lamb shift that we will ignore in this work. The atom/cavity coupling constant $g_{i j}$ represents how well the atom is coupled to the cavity. Yet again, we have ignored the finite $\mathrm{PhC}$ lifetime $\tau$, so we will stay well away from the $\omega_{b e}=\omega_{n m}$ limit, and $\beta_{k l m n}$ will not diverge.

In Eq. (29b), when $\omega_{b e}<\omega_{n m}$ the $n-m$ transition lies in the allowed propagating frequency region of the PhC. Then $\bar{\gamma}_{k l m n}$ can be thought of as a spontaneous decay rate modified by the structured vacuum near the PBE. For the opposite limit $\omega_{b e}>\omega_{n m}$ the frequency $\omega_{n m}$ lies completely in the PBG (nonpropagating photon region). The modified decay ceases and $\bar{\gamma}_{k l m n}$ acts instead as a dispersive term -i.e., the well-known frequency shifts induced by the PBE [7].

\section{THREE-LEVEL 'FIT’ ATOM IN A PHOTONIC CRYSTAL CAVITY}

Specializing Eqs. (27) to the three-level atomic system of Fig. 1 [where the two excited states are very closelyspaced ( $\omega_{31} \approx \omega_{21}$ ), each excited state is dipole-coupled to a common ground state, and the single external pump field is tuned near these excited states], and using the slowly-varying density matix elements defined by $r_{11}=1-r_{22}-r_{33}, r_{22}=\rho_{22}, r_{33}=\rho_{33}, r_{32}=\rho_{32}, r_{21}=\rho_{21} e^{i \omega_{p} t}, r_{31}=\rho_{31} e^{i \omega_{p} t}$, we find what we will call the FIT equations [1]

$$
\begin{aligned}
& \frac{d r_{31}}{d t}=-\left[\bar{\Gamma}_{3}-i\left(\Delta_{p}-\omega_{32}\right)\right] r_{31}-\bar{\Gamma}_{32} r_{21}+\Omega_{2} r_{32}+2 \Omega_{3} r_{33}+\Omega_{3} r_{22}-\Omega_{3}, \\
& \frac{d r_{21}}{d t}=-\left(\bar{\Gamma}_{2}-i \Delta_{p}\right) r_{21}-\bar{\Gamma}_{23} r_{31}+\Omega_{3} r_{23}+\Omega_{2} r_{33}+2 \Omega_{2} r_{22}-\Omega_{2}, \\
& \frac{d r_{32}}{d t}=-\left(\bar{\Gamma}_{3}+\bar{\Gamma}_{2}^{*}+i \omega_{32}\right) r_{32}-\Omega_{2}^{*} r_{31}-\Omega_{3} r_{12}-\bar{\Gamma}_{23}^{*} r_{33}-\bar{\Gamma}_{32} r_{22},
\end{aligned}
$$




$$
\begin{aligned}
& \frac{d r_{33}}{d t}=-\left(\bar{\Gamma}_{3}+\bar{\Gamma}_{3}^{*}\right) r_{33}-\Omega_{3}^{*} r_{31}-\Omega_{3} r_{13}-\bar{\Gamma}_{32}^{*} r_{32}-\bar{\Gamma}_{32} r_{23}, \\
& \frac{d r_{22}}{d t}=-\left(\bar{\Gamma}_{2}+\bar{\Gamma}_{2}^{*}\right) r_{22}-\Omega_{2}^{*} r_{21}-\Omega_{2} r_{12}-\bar{\Gamma}_{23} r_{32}-\bar{\Gamma}_{23}^{*} r_{23},
\end{aligned}
$$

plus the complex conjugates of the above. Here, $\bar{\Gamma}_{3}=\bar{\Gamma}_{3113}, \bar{\Gamma}_{2}=\bar{\Gamma}_{2112}, \bar{\Gamma}_{32}=\bar{\Gamma}_{3112}, \bar{\Gamma}_{23}=\bar{\Gamma}_{2113}, \Delta_{p}=\omega_{p}-\omega_{21}$, and the pump field Rabi frequencies are $\Omega_{m}=\frac{\underline{d}_{m 1} \bullet \underline{\varepsilon}_{p}}{\hbar} \mathcal{E}_{p}, m=2,3$. With the damping/frequency-shift terms defined as above [Eqs. (28) to (31)], the steady-state effects of the cavity field are automatically included as additional damping terms. The cavity field Rabi frequencies themselves are given in steady state by

$$
\Omega_{c m}=g_{m 1}\left\langle\hat{\alpha}_{c}\right\rangle=\frac{g_{m 1}\left(g_{21}^{*} r_{21}+g_{31}^{*} r_{31}\right)}{\left[\bar{K}_{c}\left(\delta_{c}\right)+i\left(\Delta_{c}-\Delta_{p}\right)\right]}, m=2,3,
$$

where $\hat{\alpha}_{c}(t)=\hat{a}_{c}(t) e^{i \omega_{p} t}$ is the slowly-varying amplitude of the cavity field operator and $\Delta_{c}=\omega_{c}-\omega_{21}$.

Specifically, for this FIT atom, the damping/frequency-shift terms are given by:

$$
\begin{aligned}
& \bar{\gamma}_{3}=\left\{\begin{array}{rr}
-i \beta_{3113}=-i \frac{\beta}{\sqrt{\left|\delta_{g}-\omega_{32}\right|}} \equiv-i \beta_{3}, & \omega_{b e}>\omega_{31} \\
\beta_{3}, & \omega_{b e}<\omega_{31}
\end{array},\right. \\
& \bar{\gamma}_{2}=\left\{\begin{array}{rr}
-i \beta_{2112} \approx-i \frac{\beta}{\sqrt{\left|\delta_{g}\right|} \equiv-i \beta_{2},}, \omega_{b e}>\omega_{21} \\
\beta_{2}, \omega_{b e}<\omega_{21}
\end{array},\right. \\
& \bar{\gamma}_{32}=\left\{\begin{array}{rr}
-i \beta_{3112}=-i \frac{\beta}{\sqrt{\left|\delta_{g}\right|}} \equiv-i \beta_{2}, & \omega_{b e}>\omega_{21} \\
\beta_{2}, & \omega_{b e}<\omega_{21}
\end{array}\right. \\
& \bar{\gamma}_{23}=\left\{\begin{array}{c}
-i \beta_{2113}=-i \frac{\beta}{\sqrt{\left|\delta_{g}-\omega_{32}\right|}} \equiv-i \beta_{3}, \omega_{b e}>\omega_{31} \\
\beta_{3}, \omega_{b e}<\omega_{31}
\end{array},\right. \\
& \bar{\gamma}_{c_{n 11 m}}=\frac{g^{2}}{\beta_{c} / \sqrt{\left|\delta_{g}-\Delta_{c}\right|}+i\left(\Delta_{c}-\Delta_{p}\right)}, \omega_{b e}<\omega_{c}
\end{aligned}
$$

where $\delta_{g}=\omega_{b e}-\omega_{21}$, and we have taken the two transition dipole moments $\vec{d}_{12}$ and $\vec{d}_{13}$ to be parallel, equal, and real, and we have taken $\omega_{21} \approx \omega_{31} \approx \omega_{p} \approx \omega_{c} \approx \omega_{b e}$ (unless they are subtracted from one another). With these approximations, we may also say: $g_{21} \approx g_{31}=g=g^{*}$ and $\Omega_{2}=\Omega_{3}=\Omega_{p}=\Omega_{p}^{*}$. 


\section{WEAK FIELD LIMIT}

We observe that for weak pumping, in which we set $r_{22}=r_{33}=r_{32} \approx 0$, the density matrix components $r_{21}$ and $r_{31}$ form a closed set of coupled equations (coupled by the off-diagonal decay/frequency-shift terms $\bar{\Gamma}_{32}$ and $\bar{\Gamma}_{23}$ ).

Solving these equations in steady state allows us to determine the steady-state susceptibility (which is proportional to the resonance fluorescence from the system) as

$$
\chi \propto-2 \operatorname{Re}\left[r_{21}+r_{31}\right] .
$$

In Fig. 3, we plot this susceptibility versus pump detuning (in units of $\omega_{32}$ ) in free space (i.e., we set $\left.\bar{\gamma}_{2}=\bar{\gamma}_{3}=\bar{\gamma}_{23}=\bar{\gamma}_{32}=0.05 \omega_{32}\right)$. It is hard to tell in this weak-field limit, but there is an exact zero in each of the curves at $\Delta_{p}=\omega_{32} / 2$ due to quantum interference $\left[\chi \propto\left(2 \Delta_{p}-\omega_{32}\right)^{2}\right]$. The classical interference between the pump field and the $180^{\circ}$-out-of-phase cavity field produces the deep dip at $\Delta_{p}=\Delta_{c}=0$ (dip proportional to the cavity damping constant $\beta_{c}$, where a larger decay constant implies a worse cavity and a cavity field that isn't a perfect $180^{\circ}$ out of phase).

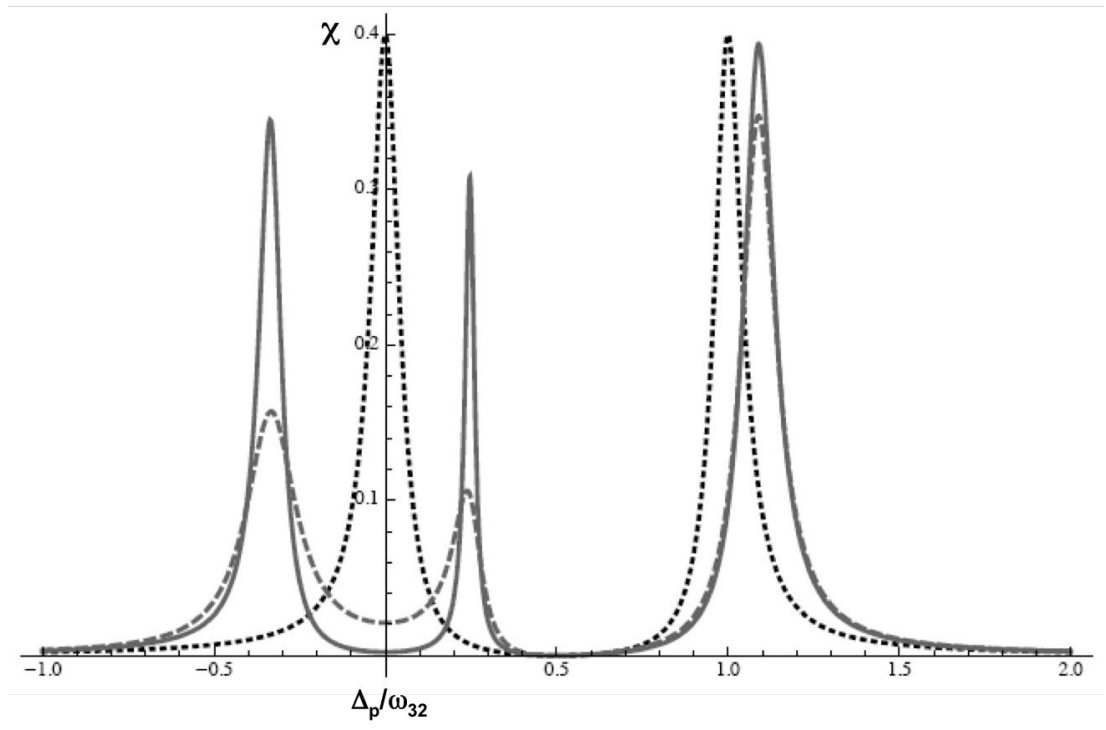

FIG. 3. Free-space $\left(\bar{\gamma}_{2}=\bar{\gamma}_{3}=\bar{\gamma}_{23}=\bar{\gamma}_{32}=0.05 \omega_{32}\right)$ susceptibility versus pump detuning. The dotted curve is for no cavity $(g=0)$. The solid and dashed curves are in a cavity $\left(g=0.3 \omega_{32}, \Delta_{\mathrm{c}}=\Delta_{\mathrm{p}}=0\right)$ with damping constants of $\beta_{c}=0.01 \omega_{32}$ and $\beta_{c}=0.1 \omega_{32}$, respectively.

In Fig. 4, we put our quantized system in a $\mathrm{PhC}$ with a band edge frequency below the 2-to- 1 transition frequency $\left(\omega_{b e}<\omega_{21}\right)$. The cavity has coupling constant and frequency given by $g=0.3 \omega_{32}, \Delta_{\mathrm{c}}=\Delta_{\mathrm{p}}=0$, and is taken to be relatively bad $\left(\beta_{c}=0.1 \omega_{32}\right)$. The dotted curve is for a system in a cavity in free space with

$\bar{\gamma}_{2}=\bar{\gamma}_{3}=\bar{\gamma}_{23}=\bar{\gamma}_{32}=0.05 \omega_{32}$. The solid and dashed curves are for a system in a PhC cavity with $\beta=0.05 \omega_{32}$, and $\delta_{g}=-0.6$ and $\delta_{g}=-0.1$, respectively. Note that when the pump frequency is tuned within the bandgap $\left(\Delta_{p}<\delta_{g}\right)$, the pump will not propagate in the $\mathrm{PhC}$ medium and the susceptibility is zero. Also note that decreasing the magnitude of $\delta_{g}$ increases the effective cavity damping constant $\beta_{c} / \sqrt{\left|\delta_{g}\right|}$, thus decreasing the classical interference dip at $\Delta_{\mathrm{c}}=\Delta_{\mathrm{p}}=0$.

In Fig. 5 we put our quantized system in a $\mathrm{PhC}$ with a band edge frequency between the 2-to-1 and 3-to- 1 transition frequencies $\left(\omega_{21}<\omega_{b e}<\omega_{31}\right)$. Once again the cavity has coupling constant and frequency given by 
$g=0.3 \omega_{32}, \Delta_{\mathrm{c}}=\Delta_{\mathrm{p}}=0.2$ (we have moved the cavity frequency so that $\omega_{b e}<\omega_{c}$ ), and is taken to be relatively bad $\left(\beta_{c}=0.1 \omega_{32}\right)$. The dotted curve is again for a system in a cavity in free space with $\bar{\gamma}_{2}=\bar{\gamma}_{3}=\bar{\gamma}_{23}=\bar{\gamma}_{32}=0.05 \omega_{32}$. The solid curve is for a system in a PhC cavity with $\beta=0.05 \omega_{32}$ and $\delta_{g}=0.1$. The pump again turns on when it exits the bandgap (when $\Delta_{p}>\delta_{g}$ ). Note that even though one of the transtion decay routes is turned off, there is still a quantum interference zero at $\Delta_{p}=\omega_{32} / 2$, but now $\chi \propto\left(2 \Delta_{p}-\omega_{32}\right)$ instead of the square. This 'weaker' quantum interference persists due to the fact that even though $\bar{\gamma}_{32}$ has become a frequency shift (pure imaginary), $\bar{\gamma}_{23}$ (pure real) still couples the two dipole moments off-diagonally.

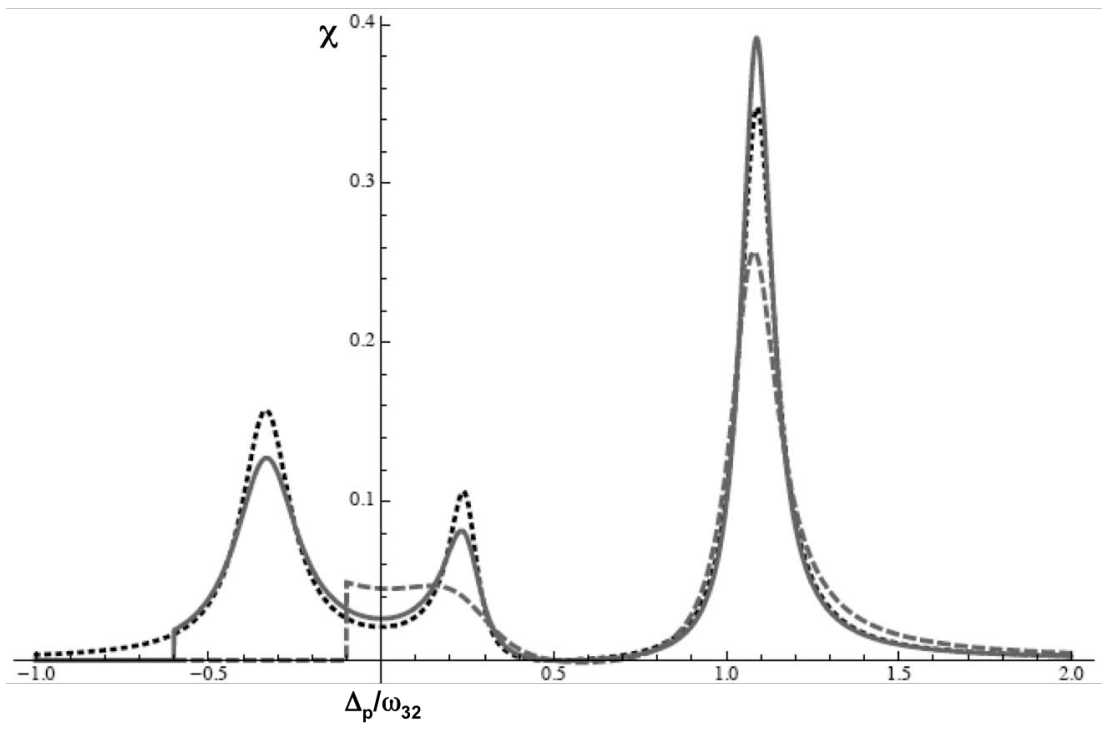

FIG. 4. Susceptibility versus pump detuning for quantized system in a cavity with $g=0.3 \omega_{32}, \Delta_{c}=0$, and $\beta_{c}=0.1 \omega_{32}$. The dotted curve is for a system in a cavity in free space with $\bar{\gamma}_{2}=\bar{\gamma}_{3}=\bar{\gamma}_{23}=\bar{\gamma}_{32}=0.05 \omega_{32}$. The solid and dashed curves are for a system in a PhC cavity with $\beta=0.05 \omega_{32}$, and $\delta_{g}=-0.6$ and $\delta_{g}=-0.1$, respectively.

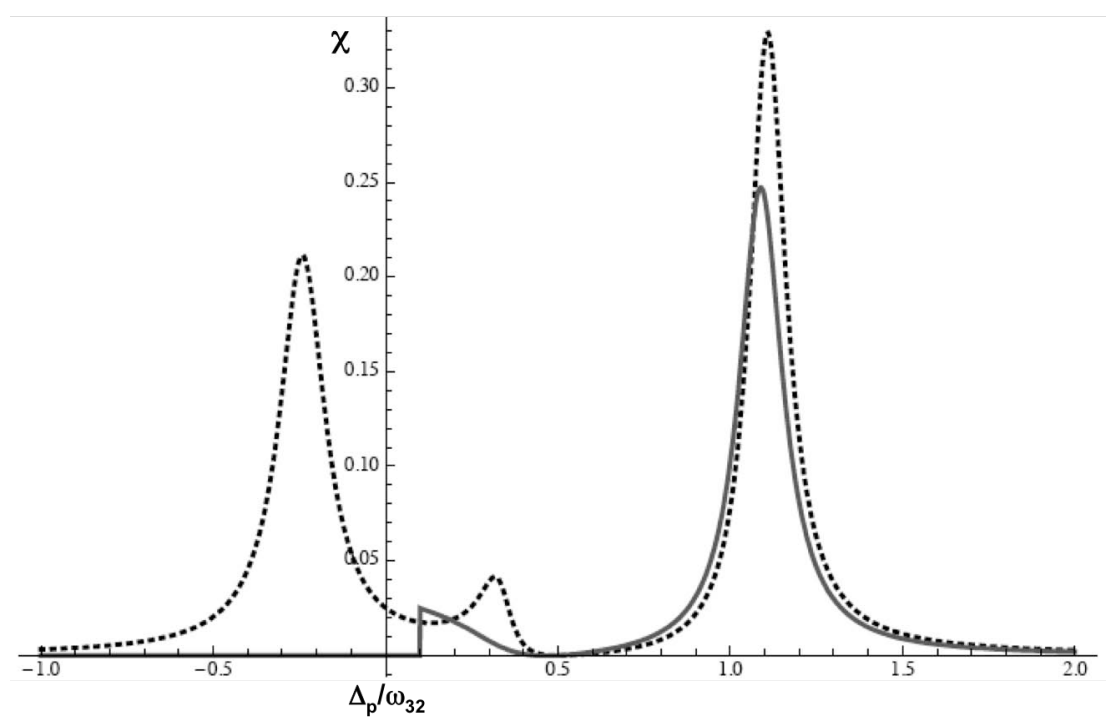

FIG. 5. Susceptibility versus pump detuning for quantized system in a cavity with $g=0.3 \omega_{32}, \Delta_{\mathrm{c}}=\Delta_{\mathrm{p}}=0.2$, and $\beta_{c}=0.1 \omega_{32}$. The dotted curve is for a system in a cavity in free space with $\bar{\gamma}_{2}=\bar{\gamma}_{3}=\bar{\gamma}_{23}=\bar{\gamma}_{32}=0.05 \omega_{32}$. The solid curve is for a system in a PhC cavity with $\beta=0.05 \omega_{32}$ and $\delta_{g}=0.1$. 
Finally, in Fig. 6, we put our quantized system in a $\mathrm{PhC}$ with a band edge frequency greater than the 3-to-1 transition frequency $\left(\omega_{b e}>\omega_{31}\right)$. The cavity has coupling constant and frequency given by $g=0.3 \omega_{32}, \Delta_{c}=\Delta_{p}=1.2$ (once again moving the cavity frequency so that $\omega_{b e}<\omega_{c}$ ), and is taken to be relatively bad $\left(\beta_{c}=0.1 \omega_{32}\right)$. The dotted curve is again for a system in a cavity in free space with $\bar{\gamma}_{2}=\bar{\gamma}_{3}=\bar{\gamma}_{23}=\bar{\gamma}_{32}=0.05 \omega_{32}$. The solid curve is for a system in a PhC cavity with $\beta=0.05 \omega_{32}$ and $\delta_{g}=1.1$. The pump again turns on when it exits the bandgap (when $\Delta_{p}>\delta_{g}$ ). We note here that if we were to plot the suceptibility for the case in which there is no cavity in the PhC, the susceptibility would be identically zero $\left(\chi \propto g^{2}\right)$ because both transitions are in the bandgap. Therefore, the presence of the cavity splits the upper level, pushing one level into the bandgap and one of of the gap. This allows for a substantial resonance fluorescence in spite of the fact that both dipole transistions are blocked by the $\mathrm{PhC}$ bandgap. This is because we have taken $\omega_{c}>\omega_{b e}$, in order to avoid a cavity with no real decay constant.

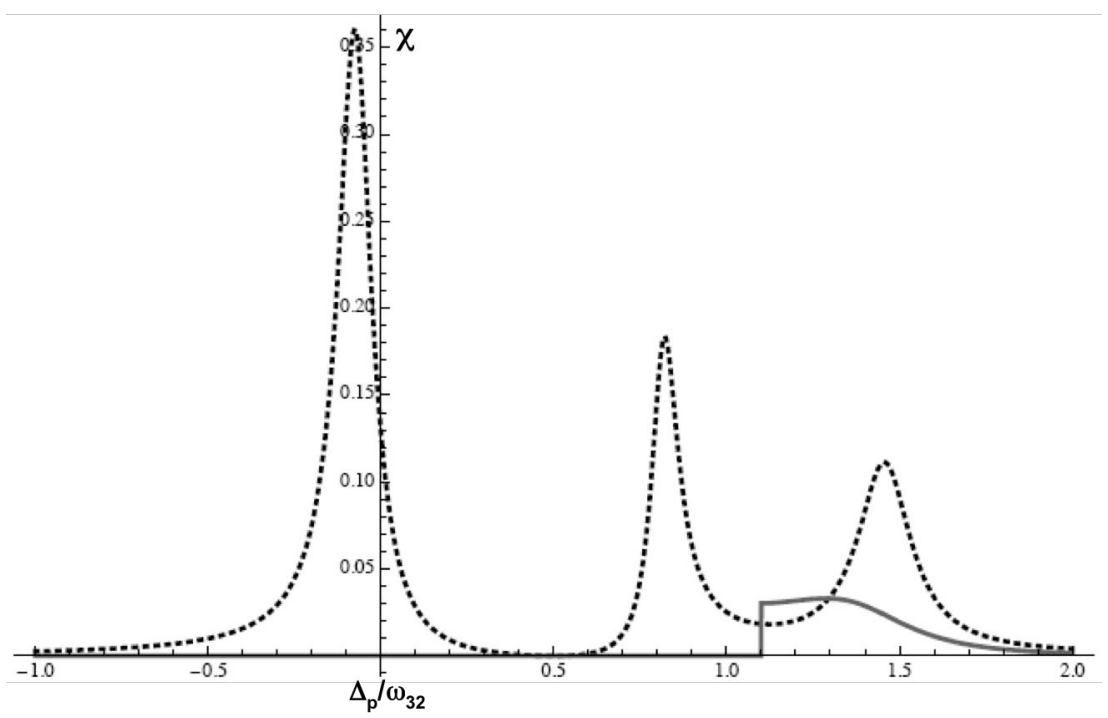

FIG. 6. Susceptibility versus pump detuning for quantized system in a cavity with $g=0.3 \omega_{32}, \Delta_{\mathrm{c}}=\Delta_{\mathrm{p}}=1.2$, and $\beta_{c}=0.1 \omega_{32}$. The dotted curve is for a system in a cavity in free space with $\bar{\gamma}_{2}=\bar{\gamma}_{3}=\bar{\gamma}_{23}=\bar{\gamma}_{32}=0.05 \omega_{32}$. The solid curve is for a system in a PhC cavity with $\beta=0.05 \omega_{32}$, and $\delta_{g}=1.1$.

\section{REFERENCES}

[1] D. A. Cardimona, M. G. Raymer, and C. R. Stroud, Jr., J. Phys. B 15, 55 (1982).

[2] S. E. Harris, Phys. Rev. Lett. 62, 1033 (1989).

[3] D. A. Cardimona, P. M. Alsing, H. Mozer, and C. Rhodes, Phys. Rev. A 79, 063817 (2009).

[4] P. M. Alsing, D. A. Cardimona, and D. H. Huang, Phys. Rev. A 76, 043802 (2007).

[5] S. John and J. Wang, Phys. Rev. Lett. 64, 2418 (1990); S. John and T. Quang, Phys. Rev. A 50, 1764 (1994); Phys. Rev. Lett. 74, 3419 (1995); N. Vats and S. John, Phys. Rev. A 58, 4168 (1998).

[6] A. Yariv and P. Yeh, Optical Waves in Crystals (Wiley, New York, 1984).

[7] G. Kofman, G. Kurizki, and B. Sherman, J. Mod. Opt. 41, 353 (1994). 


\section{DISTRIBUTION LIST}

DTIC/OCP

8725 John J. Kingman Rd, Suite 0944

Ft Belvoir, VA 22060-6218 1 cy

AFRL/RVIL

Kirtland AFB, NM 87117-5776 2 cys

Official Record Copy

AFRL/RVSS/David Cardimona $\quad 1$ cy 